\title{
CURVATURE PROPERTIES OF A SPECIAL TYPE OF PURE RADIATION METRICS
}

\begin{abstract}
ABSOS ALI SHAIKH*1, HARADHAN KUNDU², MUSAVVIR ALI ${ }^{3}$ AND ZAFAR AHSAN ${ }^{4}$
Abstract. A spacetime denotes a pure radiation field if its energy momentum tensor represents a situation in which all the energy is transported in one direction with the speed of light. In 1989, Wils and later in 1997 Ludwig and Edgar studied the physical properties of pure radiation metrics, which are conformally related to a vacuum spacetime. In the present paper we investigate the curvature properties of special type of pure radiation metrics presented by Ludwig and Edgar. It is shown that such a pure radiation spacetime is semisymmetric, Ricci simple, $R$-space by Venzi and its Ricci tensor is Riemann compatible. It is also proved that its conformal curvature 2-forms and Ricci 1-forms are recurrent. We also present a pure radiation type metric and evaluate its curvature properties along with the form of its energy momentum tensor. It is interesting to note that such pure radiation type metric is $\operatorname{Ein}(3)$ and 3-quasi-Einstein. We also find out the sufficient conditions for which this metric represents a generalized pp-wave, pure radiation and perfect fluid. Finally we made a comparison between the curvature properties of Ludwig and Edgar's pure radiation metric and pp-wave metrics.
\end{abstract}

\section{Introduction}

We consider a smooth connected semi-Riemannian manifold $\left(M^{n}, g\right)$ with $n \geq 3$ (this condition is assumed throughout the paper) equipped with the semi-Riemannian metric $g$ with signature $(p, n-p)$ and the Levi-Civita connection $\nabla$. We note that $M$ is Riemannian if $p=0$ or $n$ and $M$ is Lorentzian if $p=1$ or $n-1$. Let us consider $R, \mathcal{R}, S, \mathcal{S}$ and $\kappa$ respectively be the Riemann-Christoffel curvature tensor of type $(0,4)$, the Riemann-Christoffel curvature tensor of type $(1,3)$, the Ricci tensor of type $(0,2)$, the Ricci tensor of type $(1,1)$ and the scalar curvature of $M$.

A spacetime is a connected 4-dimensional Lorentzian manifold and it presents a pure radiation if its energy momentum tensor $T$ is of the form

$$
T=\rho \eta \otimes \eta
$$

where $\eta$ is a null vector and $\rho$ is the radiation density. Such a spacetime describes some kinds of field that propagates at the speed of light and it could represent a null electromagnetic field. It could also

Date: March 11, 2022.

2010 Mathematics Subject Classification. 53B20, 53B25, 53B30, 53B50, 53C15, 53C25, 53C35, 83C15.

Key words and phrases. pure radiation metric, pp-wave metric, generalized pp-wave metric, Einstein field equation, Weyl conformal curvature tensor, semisymmetric type curvature conditions, pseudosymmetric type curvature conditions, quasi-Einstein manifold. 
represent an incoherent beam of photons or some kinds of massless neutrino fields. On the other hand a spacetime presents a perfect fluid spacetime if its energy-momentum tensor is of the form

$$
T=(\rho+p) \eta \otimes \eta+p g
$$

where $\rho$ is the energy density, $p$ is the isotropic pressure and $\eta$ is the four-velocity of the fluid. Thus a pure radiation source can be considered as a limiting case of a pressureless perfect fluid with null four-velocity. For this reason a pure radiation source is sometimes referred as "null dust".

In [59] Wils investigated homogeneous and conformally Ricci flat solutions of Einstein's field equations for pure radiation case. Later in 1997, Ludwig and Edgar [20] obtained exhausted class of conformally Ricci flat pure radiation solutions of Einstein's field equations. The line element of conformally Ricci flat pure radiation spacetime in $\left(x^{1} ; x^{2} ; x^{3} ; x^{4}\right)=(u ; r ; x ; y)$-coordinates with $x>0$ is given by [20]

$$
d s^{2}=\left(-2 x W^{\circ}-8 \tilde{P}^{2} \frac{r^{2}}{x^{2}}\right) d u^{2}+2 d u d r-\frac{4 r}{x} d u d x-\frac{1}{8 \tilde{P}^{2}}\left(d x^{2}+d y^{2}\right),
$$

where $\tilde{P}$ is an arbitrary non-zero constant and $W^{\circ}$ is an arbitrary function of the three non-radial coordinates $u, x$ and $y$. For simplicity of symbols we write the metric as

$$
d s^{2}=\left(x w-p^{2} \frac{r^{2}}{x^{2}}\right) d u^{2}+2 d u d r-\frac{4 r}{x} d u d x-\frac{1}{p^{2}}\left(d x^{2}+d y^{2}\right),
$$

where $w=w(u, x, y)=-2 W^{\circ}$ and $p=2 \sqrt{2} \tilde{P}=$ const.

Now in $(u ; r ; x ; y)$-coordinates with $x>0$, we consider the following metric

$$
d s^{2}=\left(x w+a \frac{r^{2}}{x^{2}}\right) d u^{2}+2 d u d r+\frac{2 b r}{x} d u d x+f\left(d x^{2}+d y^{2}\right),
$$

where $a_{1}, a_{2}$ are arbitrary non-zero constants and $f$ is a nowhere vanishing function of $x$ and $y$. We note that if $a=-p^{2}, b=-2$ and $f \equiv-\frac{1}{p^{2}}$, then the metric (1.4) reduces to pure radiation metric (1.3). Hence we call the metric (1.4) as pure radiation type metric. Again if $w(u, x, y)=\frac{-2 h(u, x, y)}{x}$, $a=b=0$ and $f(x, y)=-\frac{1}{2} F(x, y)$, then the metric (1.4) reduces to generalized pp-wave metric ([29], [35], 50]), given by,

$$
d s^{2}=-2 h(u, x, y) d u^{2}+2 d u d r-\frac{1}{2} F\left(d x^{2}+d y^{2}\right) .
$$

On the other hand if $w(u, x, y)=\frac{h(u, x, y)}{x}, a=b=0$ and $f \equiv 1$, then the metric (1.4) reduces to pp-wave metric ([3], [50]), given by,

$$
d s^{2}=h(u, x, y) d u^{2}+2 d u d r+\left(d x^{2}+d y^{2}\right)
$$


The physical properties of the pure radiation metric (1.3) are well known and we refer the reader to see [20] and [59]. In the literature of differential geometry there are many curvature restricted geometric structures on a semi-Riemannian manifold, such as locally symmetric manifold [4], semisymmetric manifold ([4], [51], [52], [53]), recurrent manifold ([30], [31], [32], [58]), pseudosymmetric manifold ([1], [8] and also references therein) etc. The main object of the present paper is to investigate such kinds of geometric structures admitted by the pure radiation metric (1.3). It is noteworthy to mention that the metric (1.3) is neither locally symmetric nor conformally symmetric but semisymmetric and hence Ricci semisymmetric, conformally semisymmetric and projective semisymmetric. It is also shown that the pure radiation metric (1.3) is Ricci simple, weakly Ricci symmetric, weakly cyclic Ricci symmetric, $R$-space by Venzi and its curvature 2-forms, Ricci 1-forms and conformal curvature 2-forms are recurrent. Again the spacetime satisfies the semisymmetric type conditions $C \cdot R=0, C \cdot C=0$, $C \cdot S=0, Q(S, R)=0, Q(S, C)=0, P \cdot R=P \cdot C=0$ and also satisfies the pseudosymmetric type conditions $P \cdot P=-\frac{1}{3} Q(S, P)$. It is shown that its energy momentum tensor $T$ is semisymmetric and it is Codazzi type (resp., cyclic parallel or covariantly constant) if $w_{33}+w_{44}$ is independent of $x$ and $y$ (resp., constant or zero), where $w_{i j}$ denotes the covariant derivative with respect to $x^{i}$ and $x^{j}$.

The paper is organized as follows. Section 2 deals with the preliminaries. In section 3 we compute the components of various tensors of the metric (1.3) and we state the main results on the geometric structures admitted by pure radiation metric (1.3). Section 4 deals with the curvature properties of pure radiation type metric (1.4). It is shown that such metric is Ein(3) and 3-quasi-Einstein. We also obtain the conditions for which the metric is 2-quasi-Einstein, Ricci generalized pseudosymmetric and manifold of vanishing scalar curvature. Finally, we made a comparison (similarities and dissimilarities) between pure radiation metric and pp-wave metric. It is interesting to mention that both are semisymmetric and weakly Ricci symmetric, but generalized pp-wave metric is Ricci recurrent whereas pure radiation metric is not so.

\section{Curvature Restricted Geometric Structures}

It is wellknown that a curvature restricted geometric structure is a geometric structure on a semiRiemannian manifold $M$ obtained by imposing a restriction on its curvature tensors by means of covariant derivatives of first order or higher orders. We will now explain some useful notations and definitions of various curvature restricted geometric structures.

For two symmetric $(0,2)$-tensors $A$ and $E$, their Kulkarni-Nomizu product $A \wedge E$ is defined as (see e.g. [12, [17]):

$$
\begin{aligned}
(A \wedge E)\left(X_{1}, X_{2}, X_{3}, X_{4}\right) & =A\left(X_{1}, X_{4}\right) E\left(X_{2}, X_{3}\right)+A\left(X_{2}, X_{3}\right) E\left(X_{1}, X_{4}\right) \\
& -A\left(X_{1}, X_{3}\right) E\left(X_{2}, X_{4}\right)-A\left(X_{2}, X_{4}\right) E\left(X_{1}, X_{3}\right),
\end{aligned}
$$


where $X_{1}, X_{2}, X_{3}, X_{4} \in \chi(M)$, the Lie algebra of all smooth vector fields on $M$. Throughout the paper we will consider $X, Y, X_{1}, X_{2}, \cdots \in \chi(M)$.

Again for a symmetric $(0,2)$-tensor $A$, we get an endomorphism $\mathcal{A}$ defined by $g(\mathcal{A} X, Y)=A(X, Y)$. Then its $k$-th level tensor $A^{k}$ of type $(0,2)$ is given by

$$
A^{k}(X, Y)=A\left(\mathcal{A}^{k-1} X, Y\right),
$$

where $\mathcal{A}^{k-1}$ is the endomorphism corresponding to $A^{k-1}$.

In terms of Kulkarni-Nomizu product the conformal curvature tensor $C$, concircular curvature tensor $W$, conharmonic curvature tensor $K([18],[60])$ and the Gaussian curvature tensor $\mathfrak{G}$ can respectively be expressed as

$$
\begin{aligned}
C & =R-\frac{1}{n-2}(g \wedge S)+\frac{r}{2(n-2)(n-1)}(g \wedge g), \\
W & =R-\frac{r}{2 n(n-1)}(g \wedge g), \\
K & =R-\frac{1}{n-2}(g \wedge S), \\
\mathfrak{G} & =\frac{1}{2}(g \wedge g) .
\end{aligned}
$$

Again the projective curvature tensor $P$ is given by

$$
P\left(X_{1}, X_{2}, X_{3}, X_{4}\right)=R\left(X_{1}, X_{2}, X_{3}, X_{4}\right)-\frac{1}{n-1}\left[g\left(X_{1}, X_{4}\right) S\left(X_{2}, X_{3}\right)-g\left(X_{2}, X_{4}\right) S\left(X_{1}, X_{3}\right)\right] .
$$

For a symmetric $(0,2)$-tensor $A,(0,4)$-tensor $D$ and a $(0, k)$-tensor $H, k \geq 1$, one can define two $(0, k+2)$-tensors $D \cdot H$ and $Q(A, H)$ respectively as follows (see [9], [10], [14], [36], [38] and also references therein):

$$
D \cdot H\left(X_{1}, X_{2}, \cdots, X_{k}, X, Y\right)=-H\left(\mathcal{D}(X, Y) X_{1}, X_{2}, \cdots, X_{k}\right)-\cdots-H\left(X_{1}, X_{2}, \cdots, \mathcal{D}(X, Y) X_{k}\right)
$$

and

$$
\begin{aligned}
Q(A, H)\left(X_{1}, X_{2}, \ldots, X_{k}, X, Y\right) & =A\left(X, X_{1}\right) H\left(Y, X_{2}, \cdots, X_{k}\right)+\cdots+A\left(X, X_{k}\right) H\left(X_{1}, X_{2}, \cdots, Y\right) \\
& -A\left(Y, X_{1}\right) H\left(X, X_{2}, \cdots, X_{k}\right)-\cdots-A\left(Y, X_{k}\right) H\left(X_{1}, X_{2}, \cdots, X\right),
\end{aligned}
$$

where $\mathcal{D}$ is the corresponding (1,3)-tensor of $D$, given by $D\left(X_{1}, X_{2}, X_{3}, X_{4}\right)=g\left(\mathcal{D}\left(X_{1}, X_{2}\right) X_{3}, X_{4}\right)$.

Definition 2.1. A semi-Riemannian manifold $M$ is said to be $H$-symmetric ([4], [5]) if $\nabla H=0$. In particular if $H=R$ (resp., $S$ and $C$ ), then the manifold is called locally symmetric (resp., Ricci symmetric and conformally symmetric). 
Definition 2.2. A symmetric (0,2)-tensor $E$ on $M$ is said to be cyclic parallel (resp, Codazzi type) (see, [15], [16] and references therein) if

$$
\begin{gathered}
\left(\nabla_{X_{1}} E\right)\left(X_{2}, X_{3}\right)=\left(\nabla_{X_{2}} E\right)\left(X_{1}, X_{3}\right) \\
\left(\text { resp., }\left(\nabla_{X_{1}} E\right)\left(X_{2}, X_{3}\right)+\left(\nabla_{X_{2}} E\right)\left(X_{3}, X_{1}\right)+\left(\nabla_{X_{3}} E\right)\left(X_{1}, X_{2}\right)=0\right) .
\end{gathered}
$$

Definition 2.3. (11, [5], 8], 38], 41], 42, [51]) A semi-Riemannian manifold $M$ is said to be $H$ semisymmetric type if $D \cdot H=0$ and it is said to be $H$-pseudosymmetric type if $\left(\sum_{i=1}^{k} c_{i} D_{i}\right) \cdot H=0$ for some scalars $c_{i}$ 's, where $D$ and each $D_{i}, i=1, \ldots, k,(k \geq 2)$, are $(0,4)$ curvature tensors.

Definition 2.4. A semi-Riemannian manifold $M$ is said to be Einstein if its Ricci tensor is a scalar multiple of the metric tensor g. Again $M$ is called quasi-Einstein (resp., 2-quasi-Einstein and 3-quasiEinstein) if at each point of $M, \operatorname{rank}(S-\alpha g) \leq 1$ (resp., $\leq 2$ and $\leq 3$ ) for a scalar $\alpha$. In particular, if $\alpha=0$, then a quasi-Einstein manifold is called Ricci simple.

We note that Som-Raychaudhuri metric [40] and Robinson-Trautman metric [34] are 2-quasi-Einstein whereas Gödel metric [15] is Ricci simple.

Definition 2.5. (2], [39]) A semi-Riemannian manifold $M$ is said to be Ein(2), Ein(3) and Ein(4) respectively if

$$
\begin{gathered}
S^{2}+\lambda_{1} S+\lambda_{2} g=0 \\
S^{3}+\lambda_{3} S^{2}+\lambda_{4} S+\lambda_{5} g=0 \text { and } \\
S^{4}+\lambda_{6} S^{3}+\lambda_{7} S^{2}+\lambda_{8} S+\lambda_{9} g=0
\end{gathered}
$$

holds for some scalars $\lambda_{i}, 1 \leq i \leq 9$.

Definition 2.6. Let $D$ be a (0,4)-tensor and $E$ be a symmetric $(0,2)$-tensor on $M$. Then $E$ is said to be D-compatible ([11], [21], [22]) if

$$
D\left(\mathcal{E} X_{1}, X, X_{2}, X_{3}\right)+D\left(\mathcal{E} X_{2}, X, X_{3}, X_{1}\right)+D\left(\mathcal{E} X_{3}, X, X_{1}, X_{2}\right)=0
$$

holds, where $\mathcal{E}$ is the endomorphism corresponding to $E$ defined as $g\left(\mathcal{E} X_{1}, X_{2}\right)=E\left(X_{1}, X_{2}\right)$. Again an 1-form $\Pi$ is said to be $D$-compatible if $\Pi \otimes \Pi$ is $D$-compatible.

Generalizing the concept of recurrent manifold ([30], 331], [32], [58]), recently Shaikh et al. [49] introduced the notion of super generalized recurrent manifold along with its characterization and existence by proper example.

Definition 2.7. A semi-Riemannian manifold $M$ is said to be super generalized recurrent manifold ([38], [44], 49]) if

$$
\nabla R=\Pi \otimes R+\Omega \otimes(S \wedge S)+\Theta \otimes(g \wedge S)+\omega \otimes(g \wedge g)
$$


holds on $\{x \in M: R \neq 0$ and any one of $S \wedge S, g \wedge S$ is non-zero at $x\}$ for some 1-forms $\Pi, \Omega, \Theta$ and $\omega$, called the associated 1-forms. Especially, if $\Omega=\Theta=\omega=0$ (resp., $\Theta=\omega=0$ and $\Omega=\omega=0$ ), then the manifold is called recurrent ([30], [31], [32], [58]) (resp., weakly generalized recurrent ([33], [47]) and hyper generalized recurrent (46], [48])) manifold.

Again as a generalization of locally symmetric manifold and recurrent manifold, Tamássy and Binh [55] introduced the notion of weakly symmetric manifolds.

Definition 2.8. Let $D$ be a (0,4)-tensor on a semi-Riemannian manifold $M$. Then $M$ is said to be weakly D-symmetric manifold ([55], [37]) if

$$
\begin{aligned}
& \nabla_{X} D\left(X_{1}, X_{2}, X_{3}, X_{4}\right)=\Pi(X) D\left(X_{1}, X_{2}, X_{3}, X_{4}\right)+\Omega\left(X_{1}\right) D\left(X_{1}, X_{2}, X_{3}, X_{4}\right) \\
& \quad+\bar{\Omega}\left(X_{2}\right) D\left(X_{1}, X, X_{3}, X_{4}\right)+\Theta\left(X_{3}\right) D\left(X_{1}, X_{2}, X, X_{4}\right)+\bar{\Theta}\left(X_{4}\right) D\left(X_{1}, X_{2}, X_{3}, X\right)
\end{aligned}
$$

holds $\forall X, X_{i} \in \chi(M)(i=1,2,3,4)$ and some 1-forms $\Pi, \Omega, \bar{\Omega}, \Theta$ and $\bar{\Theta}$ on $\left\{x \in M: R_{x} \neq 0\right\}$. Such a manifold is called as weakly D-symmetric manifold with solution $(\Pi, \Omega, \bar{\Omega}, \Theta, \bar{\Theta})$. In particular, if the solution is of the form $(2 \Pi, \Pi, \Pi, \Pi, \Pi)$, then the manifold is called Chaki D-pseudosymmetric manifold [6]. Again if the solution is of the form $(\Pi, 0,0,0,0)$ then the manifold is called D-recurrent manifold ([30], 31], 32], [58]).

Definition 2.9. Let $Z$ be a (0, 2)-tensor on a semi-Riemannian manifold $M$. Then $M$ is said to be weakly $Z$-symmetric ([56], 37]) if

$$
\left(\nabla_{X} Z\right)\left(X_{1}, X_{2}\right)=\Pi(X) Z\left(X_{1}, X_{2}\right)+\Omega\left(X_{1}\right) Z\left(X, X_{2}\right)+\Theta\left(X_{2}\right) Z\left(X_{1}, X\right)
$$

holds $\forall X, X_{1}, X_{2} \in \chi(M)$ and some 1-forms $\Pi, \Omega$ and $\Theta$ on $U_{Z}=\{x \in M: Z \neq 0$ at $x\}$. Such a manifold is called as weakly $Z$-symmetric manifold with solution $(\Pi, \Omega, \Theta)$. Especially, if the solution is of the form $(2 \Pi, \Pi, \Pi)$ then the manifold is called Chaki pseudo Z-symmetric manifold [7]. Again if the solution is of the form $(\Pi, 0,0)$ then the manifold is called Z-recurrent [27].

For details about the defining condition of weak symmetry and the interrelation between weak symmetry and Deszcz psudosymmetry, we refer the reader to see [36] and also references therein.

Definition 2.10. A Riemannian manifold $M$ is said to be weakly cyclic Ricci symmetric [45] if its Ricci tensor satisfies the condition

$$
\begin{aligned}
& \left(\nabla_{X} S\right)\left(X_{1}, X_{2}\right)+\left(\nabla_{X_{1}} S\right)\left(X, X_{2}\right)+\left(\nabla_{X_{2}} S\right)\left(X_{1}, X\right) \\
& =\Pi(X) S\left(X_{1}, X_{2}\right)+\Omega\left(X_{1}\right) S\left(X, X_{2}\right)+\Theta\left(X_{2}\right) S\left(X_{1}, X\right),
\end{aligned}
$$

for three 1-forms $\Pi, \Omega$ and $\Theta$ on $M$. Such a manifold is called weakly cyclic Ricci symmetric manifold with solution $(\Pi, \Omega, \Theta)$. 
It is noteworthy to mention that the solution of weakly cyclic Ricci symmetric structure is not always unique.

Definition 2.11. Let $D$ be a $(0,4)$ tensor and $Z$ be a $(0,2)$-tensor on $M$. Then the corresponding curvature 2-forms $\Omega_{(D) l}^{m}([2]$, [19]) are said to be recurrent if and only if ([23], [24], [25])

$$
\begin{gathered}
\left(\nabla_{X_{1}} D\right)\left(X_{2}, X_{3}, X, Y\right)+\left(\nabla_{X_{2}} D\right)\left(X_{3}, X_{1}, X, Y\right)+\left(\nabla_{X_{3}} D\right)\left(X_{1}, X_{2}, X, Y\right)= \\
\Pi\left(X_{1}\right) D\left(X_{2}, X_{3}, X, Y\right)+\Pi\left(X_{2}\right) D\left(X_{3}, X_{1}, X, Y\right)+\Pi\left(X_{3}\right) D\left(X_{1}, X_{2}, X, Y\right),
\end{gathered}
$$

and the 1-forms $\Lambda_{(Z) l}$ [54] are said to be recurrent if and only if

$$
\left(\nabla_{X_{1}} Z\right)\left(X_{2}, X\right)-\left(\nabla_{X_{2}} Z\right)\left(X_{1}, X\right)=\Pi\left(X_{1}\right) Z\left(X_{2}, X\right)-\Pi\left(X_{2}\right) Z\left(X_{1}, X\right)
$$

for an 1-form $\Pi$.

Definition 2.12. ([28], [41], [57]) Let $\mathcal{L}(M)$ be the vector space formed by all 1-forms $\Theta$ on $M$ satisfying

$$
\Theta\left(X_{1}\right) D\left(X_{2}, X_{3}, X_{4}, X_{5}\right)+\Theta\left(X_{2}\right) D\left(X_{3}, X_{1}, X_{4}, X_{5}\right)+\Theta\left(X_{3}\right) D\left(X_{1}, X_{2}, X_{4}, X_{5}\right)=0
$$

where $D$ is a $(0,4)$-tensor. Then $M$ is said to be a $D$-space by Venzi if $\operatorname{dim}[\mathcal{L}(M)] \geq 1$.

From definition of recurrency of curvature 2-forms $\Omega_{(R) l}^{m}$ and second Bianchi identity it is clear that on a semi-Riemannian manifold $\Omega_{(R) l}^{m}$ are recurrent if and only if it is a $R$ space by Venzi.

\section{Curvature properties of pure radiation metric}

The metric tensor of pure radiation metric (1.3) is given by

$$
g=\left(\begin{array}{cccc}
\left(x w-p^{2} \frac{r^{2}}{x^{2}}\right) & 1 & \frac{-2 r}{x} & 0 \\
1 & 0 & 0 & 0 \\
\frac{-2 r}{x} & 0 & -\frac{1}{p^{2}} & 0 \\
0 & 0 & 0 & -\frac{1}{p^{2}}
\end{array}\right) .
$$

Then the non-zero components (upto symmetry) of its Riemann-Christoffel curvature tensor $R$, Ricci tensor $S$, scalar curvature $\kappa$, conformal curvature tensor $C$ and projective curvature tensor $P$ are given by

$$
\begin{gathered}
R_{1313}=-\frac{w_{33} x}{2}, \quad R_{1314}=-\frac{w_{34} x}{2}, \quad R_{1414}=-\frac{w_{44} x}{2} \\
S_{11}=-\frac{1}{2} p^{2}\left(w_{33}+w_{44}\right) x, \quad \kappa=0 \\
-C_{1313}=C_{1414}=\frac{1}{4}\left(w_{33}-w_{44}\right) x, \quad C_{1314}=-\frac{w_{34} x}{2},
\end{gathered}
$$




$$
\begin{gathered}
P_{1211}=-\frac{1}{6} p^{2}\left(w_{33}+w_{44}\right) x, \quad P_{1311}=\frac{1}{3} p^{2} r\left(w_{33}+w_{44}\right), \\
P_{1313}=-\frac{1}{6}\left(2 w_{33}-w_{44}\right) x, \quad-P_{1314}=P_{1341}=-P_{1413}=P_{1431}=\frac{w_{34} x}{2}, \\
P_{1331}=\frac{w_{33} x}{2}, \quad P_{1414}=\frac{1}{6}\left(w_{33}-2 w_{44}\right) x, \quad P_{1441}=\frac{w_{44} x}{2} .
\end{gathered}
$$

Then from above it is easy to check that $R \cdot R=Q(S, R)=R \cdot C=Q(S, C)=0$.

Now the non-zero components (upto symmetry) of $\nabla R, \nabla S, \nabla C$ are given by

$$
\begin{gathered}
R_{1213,1}=\frac{p^{2} w_{33}}{2}, \quad R_{1214,1}=\frac{p^{2} w_{34}}{2}, \quad R_{1313,1}=-\frac{x^{2} w_{133}+2 p^{2} r w_{33}}{2 x}, \quad R_{1414,1}=\frac{2 p^{2} r w_{44}-x^{2} w_{144}}{2 x} \\
R_{1313,3}=\frac{1}{2}\left(w_{33}-w_{333} x\right), \quad R_{1313,4}=-\frac{w_{334} x}{2}, \quad R_{1314,1}=-\frac{w_{134} x}{2}, \quad R_{1414,3}=\frac{1}{2}\left(w_{44}-w_{344} x\right) \\
R_{1314,3}=\frac{1}{2}\left(w_{34}-w_{334} x\right), \quad R_{1314,4}=-\frac{w_{344} x}{2}, \quad R_{1334,1}=\frac{w_{34}}{2}, \quad R_{1414,4}=-\frac{w_{444} x}{2}, \quad R_{1434,1}=\frac{w_{44}}{2} \\
S_{11,1}=\frac{p^{2}\left(-x^{2} w_{144}-x^{2} w_{133}+2 p^{2} r w_{33}+2 p^{2} r w_{44}\right)}{2 x}, \\
S_{11,3}=\frac{1}{2} p^{2}\left(-w_{333} x-w_{344} x+w_{33}+w_{44}\right) \\
S_{11,4}=-\frac{1}{2} p^{2}\left(w_{334}+w_{444}\right) x, \quad S_{13,1}=\frac{1}{2} p^{2}\left(w_{33}+w_{44}\right) \\
C_{1213,1}=\frac{1}{4} p^{2}\left(w_{33}-w_{44}\right), \quad C_{1214,1}=\frac{p^{2} w_{34}}{2}, \\
C_{1313,1}=-\frac{-x^{2} w_{144}+x^{2} w_{133}+2 p^{2} r w_{33}-2 p^{2} r w_{44}}{4 x}, \\
C_{1313,3}=-C_{1414,3}=\frac{1}{4}\left(-w_{333} x+w_{344} x+w_{33}-w_{44}\right) \\
-C_{1313,4}=C_{1414,4}=\frac{1}{4}\left(w_{334}-w_{444}\right) x, \quad C_{1314,1}=-\frac{w_{134} x}{2} \\
C_{1314,3}=\frac{1}{2}\left(w_{34}-w_{334} x\right), \quad C_{1314,4}=-\frac{w_{344} x}{2}, \quad C_{1334,1}=\frac{w_{34}}{2} \\
C_{1414,1}=-\frac{1}{4 w_{144}-x^{2} w_{133}+2 p^{2} r w_{33}-2 p^{2} r w_{44}} \\
C_{1434,1}=-\frac{1}{4}\left(w_{33}-w_{44}\right)
\end{gathered}
$$

According to Einstein's field equations, the energy momentum tensor $T$ for zero cosmological constant is related to the Ricci tensor and the metric tensor as

$$
T=\frac{c^{4}}{8 \pi G}\left[S-\left(\frac{\kappa}{2}\right) g\right]
$$

where $c=$ speed of light in vacuum and $G=$ gravitational constant. Thus the non-zero components of the energy momentum tensor of the pure radiation metric (1.3) is given by:

$$
T_{11}=-\frac{c^{4}\left(p^{2} w_{33} x+p^{2} w_{44} x\right)}{16 \pi G x^{2}} .
$$


Obviously, $T=\rho(\eta \otimes \eta)$, where $\eta=\{1,0,0,0\}$ and the radiation density $\rho=T_{11}$. It is easy to check that $\|\eta\|=0$.

Now the non-zero components of covariant derivative of $T$ are given by

$$
\begin{gathered}
T_{11,1}=\frac{c^{4} p^{2}\left(-x^{2} w_{144}-x^{2} w_{133}+2 p^{2} r w_{33}+2 p^{2} r w_{44}\right)}{16 \pi G x}, \quad T_{13,1}=\frac{c^{4} p^{2}\left(w_{33}+w_{44}\right)}{16 \pi G} \\
T_{11,3}=\frac{c^{4} p^{2}\left(-w_{333} x-w_{344} x+w_{33}+w_{44}\right)}{16 \pi G}, \quad T_{11,4}=-\frac{c^{4} p^{2}\left(w_{334}+w_{444}\right) x}{16 \pi G} .
\end{gathered}
$$

From the value of the local components (presented in Section 3) of various tensors of the pure radiation metric (1.3), we can conclude that the pure radiation metric (1.3) fulfills the following curvature restricted geometric structures.

Theorem 3.1. The pure radiation metric (1.3) possesses the following curvature properties:

(i) Its Ricci tensor is neither Codazzi type nor cyclic parallel but the scalar curvature is zero and hence $R=W$ and $C=K$.

(ii) It is a $R$-space (also $C$-space, $P$-space) by Venzi for the associated 1 -form $\Pi=\{c, 0,0,0\}, c$ being arbitrary scalar. Hence the curvature 2 -forms $\Omega_{(R) l}^{m}$ are recurrent for $\Pi$ as the 1-forms of recurrency.

(iii) It is neither locally symmetric nor conformally symmetric but semisymmetric. Hence it satisfies $R \cdot S=0, R \cdot C=0$ and $R \cdot P=0$.

(iv) It satisfies the semisymmetric type condition $C \cdot R=0$ and hence $C \cdot S=0, C \cdot C=0, C \cdot P=0$ and $P \cdot S=0$.

(v) $R$ or $C$ of the space is not a scalar multiple of $S \wedge S$, but $Q(S, R)=0, Q(S, C)=0$. Hence $P \cdot R=0$ and $P \cdot C=0$, although $P \cdot \mathcal{R} \neq 0$ but $P \cdot \mathcal{S}=0$.

(vi) It is not Einstein but Ricci simple, since $S=\beta(\eta \otimes \eta)$, where $\beta=-\frac{p^{2} x\left(w_{33}+w_{44}\right)}{2}$ and $\eta=$ $\{1,0,0,0\}$ (moreover $\|\eta\|=0$ and $\nabla \eta \neq 0)$. Hence $S \wedge S=0$ and $S^{2}=0$.

(vii) Here $P \cdot P \neq 0$ but $P \cdot P=-\frac{1}{3} Q(S, P)$.

(viii) If $w_{33}+w_{44}$ is nowhere zero, then the metric is neither recurrent nor Ricci recurrent but Ricci 1-forms are recurrent with associated 1-form

$$
\left\{1,0, \frac{w_{333}+w_{344}}{w_{33}+w_{44}}, \frac{w_{334}+w_{444}}{w_{33}+w_{44}}\right\} .
$$

(ix) If $4 w_{34}^{2}+\left(w_{44}-w_{33}\right)^{2}$ is nowhere zero, then the metric is not conformally recurrent but conformal 2-forms are recurrent with associated 1-form $\Pi$, given by

$$
\begin{gathered}
\Pi_{1}=1, \quad \Pi_{2}=0 \\
\Pi_{3}=\frac{2 w_{34}\left(w_{334}+w_{444}\right)-\left(w_{44}-w_{33}\right)\left(w_{333}+w_{344}\right)}{4 w_{34}^{2}+\left(w_{44}-w_{33}\right)^{2}}
\end{gathered}
$$




$$
\Pi_{4}=\frac{2 w_{34}\left(w_{333}+w_{344}\right)+\left(w_{44}-w_{33}\right)\left(w_{334}+w_{444}\right)}{4 w_{34}^{2}+\left(w_{44}-w_{33}\right)^{2}} .
$$

(x) The general form of the compatible tensor for $R, C$ and $P$ are respectively given by

$$
\begin{gathered}
\left(\begin{array}{ccccc}
a_{(1,1)} & a_{(1,2)} & a_{(1,3)} & a_{(1,4)} \\
a_{(2,1)} & 0 & 0 & 0 & \\
a_{(3,1)} & 0 & a_{(3,3)} & a_{(3,4)} \\
a_{(4,1)} & 0 & a_{(4,3)} & a_{(3,3)}+\frac{w_{44} a_{(3,4)}}{w_{34}}-\frac{w_{33} a_{(4,3)}}{w_{34}}
\end{array}\right) \\
\left(\begin{array}{cccc}
a_{(1,1)} & a_{(1,2)} & a_{(1,3)} & a_{(1,4)} \\
a_{(2,1)} & 0 & 0 & 0 \\
a_{(3,1)} & 0 & a_{(3,3)} & a_{(3,4)} \\
a_{(4,1)} & 0 & -\frac{2 w_{34} a_{(3,3)}}{w_{44}-w_{33}}-a_{(3,4)}+\frac{2 w_{34} a_{(4,4)}}{w_{44}-w_{33}} & a_{(4,4)}
\end{array}\right) \\
a_{(1,1)} a_{(1,2)} \\
a_{(2,1)} \\
0
\end{gathered}
$$

where $a_{(i, j)}$ are arbitrary scalars.

(xi) Ricci tensor of this spacetime is not Codazzi type but is compatible for $R, C$ and $P$.

(xii) If $w_{33}+w_{44}$ is nowhere vanishing, then the metric is weakly Ricci symmetric with infinitely many solutions $(\Pi, \Omega, \Theta)$, given by

$$
\begin{gathered}
\Pi=\left\{\Pi_{1}, 0, \frac{w_{333} x+w_{344} x-w_{33}-w_{44}}{\left(w_{33}+w_{44}\right) x}, \frac{w_{334}+w_{444}}{w_{33}+w_{44}}\right\}, \\
\Omega=\left\{\Omega_{1}, 0,-\frac{1}{x}, 0\right\} \text { and } \\
\Theta=\left\{\frac{x^{2} w_{144} x^{2} w_{133}-2 p^{2} r w_{33}-2 p^{2} r w_{44}}{\left(w_{33}+w_{44}\right) x^{2}}-\Pi_{1}-\Omega_{1}, 0,-\frac{1}{x}, 0\right\},
\end{gathered}
$$

where $\Pi_{1}$ and $\Omega_{1}$ are arbitrary scalars.

(xiii) If $w_{33}+w_{44}$ is nowhere vanishing, then the metric is weakly cyclic Ricci symmetric with infinitely many solutions $(\Pi, \Omega, \Theta)$, given by

$$
\begin{gathered}
\Pi=\left\{\Pi_{1}, 0, \frac{\left(w_{333}+w_{344}\right) x-3 w_{33}-3 w_{44}}{\left(w_{33}+w_{44}\right) x}, \frac{w_{334}+w_{444}}{w_{33}+w_{44}}\right\}, \\
\Omega=\left\{\Omega_{1}, 0, \frac{\left(w_{333}+w_{344}\right) x-3 w_{33}-3 w_{44}}{\left(w_{33}+w_{44}\right) x}, \frac{w_{334}+w_{444}}{w_{33}+w_{44}}\right\} \quad \text { and }
\end{gathered}
$$




$$
\begin{aligned}
\Theta=\left\{\frac{3\left(x^{2}\left(w_{144}+w_{133}\right)-2 p^{2} r\left(w_{33}+w_{44}\right)\right)}{\left(w_{33}+w_{44}\right) x^{2}}-\Pi_{1}-\Omega_{1}, 0,\right. & \left.\frac{\left(w_{333}+w_{344}\right) x-3 w_{33}-3 w_{44}}{\left(w_{33}+w_{44}\right) x}, \frac{w_{334}+w_{444}}{w_{33}+w_{44}}\right\},
\end{aligned}
$$

where $\Pi_{1}$ and $\Omega_{1}$ are arbitrary scalars.

(xiv) It is not weakly symmetric for $R, C, P, W$ and $K$ and hence not Chaki pseudosymmetric for $R, C$ or $P$.

(xv) $\operatorname{div} R \neq 0, \operatorname{div} C \neq 0, \operatorname{div} P \neq 0$.

Now from the values of the non-zero components of $\nabla T$, we get

$$
\left.\begin{array}{l}
T_{11,1}+T_{11,1}+T_{11,1}=\frac{3 c^{4} p^{2}\left(-x^{2} w_{144}-x^{2} w_{133}+2 p^{2} r w_{33}+2 p^{2} r w_{44}\right)}{16 \pi G x}, \\
T_{11,3}+T_{13,1}+T_{31,1}=\frac{c^{4} p^{2}\left(-w_{333} x-w_{344} x+3 w_{33}+3 w_{44}\right)}{16 \pi G}, \\
T_{11,4}+T_{14,1}+T_{41,1}=-\frac{c^{4} p^{2}\left(w_{334}+w_{444}\right) x}{16 \pi G}
\end{array}\right\}
$$

and

$$
\left.\begin{array}{l}
T_{13,1}-T_{11,3}=\frac{c^{4} p^{2}\left(w_{333}+w_{344}\right) x}{16 \pi G}, \\
T_{14,1}-T_{11,4}=\frac{c^{4} p^{2}\left(w_{334}+w_{444}\right) x}{16 \pi G} .
\end{array}\right\}
$$

Again since $R \cdot S=0$ and $T$ is a linear combination of $S$ and $g$, so $R \cdot T=0$. Hence we can state the following:

Theorem 3.2. The energy-momentum tensor $T$ of the pure radiation metric (1.3) is

(i) semisymmertric i.e., $R \cdot T=0$,

(ii) Codazzi type if $w_{33}+w_{44}$ is independent of $x$ and $y$,

(iii) cyclic parallel if $w_{33}+w_{44}$ is independent of $u, x$ and $y$,

(iv) covariantly constant if $w_{33}+w_{44}=0$.

\section{Curvature properties of pure radiation type metric}

We now consider the pure radiation type metric (1.4). Its metric tensor is given by

$$
g=\left(\begin{array}{cccc}
\left(x w+a \frac{r^{2}}{x^{2}}\right) & 1 & \frac{b r}{x} & 0 \\
1 & 0 & 0 & 0 \\
\frac{b r}{x} & 0 & f & 0 \\
0 & 0 & 0 & f
\end{array}\right),
$$

where $a, b$ are arbitrary non-zero constants and $w=w(u, x, y), f=f(x, y)$ are nowhere vanishing functions. Then the non-zero components (upto symmetry) of $R, S$ and $\kappa$ are given by

$$
R_{1212}=-\frac{4 a f-b^{2}}{4 f x^{2}}, \quad R_{1213}=\frac{r\left(8 a f+b^{3}\right)}{4 f x^{3}}, \quad R_{1334}=-\frac{b^{2} f_{4} r}{4 f x^{2}}
$$




$$
\begin{gathered}
R_{1313}=-\frac{1}{4 f x^{4}} \quad\left[a b^{2} f r^{2}+2 a b f_{3} r^{2} x+2 a f_{3} r^{2} x+12 a f r^{2}-b^{4} r^{2}+b^{2} f w x^{3}\right. \\
\left.+2 b f w_{3} x^{4}+2 b f w x^{3}-f_{3} w_{3} x^{5}+f_{4} w_{4} x^{5}+2 f w_{33} x^{5}-f_{3} w x^{4}+4 f w_{3} x^{4}\right] \\
R_{1314}=-\frac{2 a b f_{4} r^{2}+2 a f_{4} r^{2}+b f w_{4} x^{3}-f_{4} w_{3} x^{4}-f_{3} w_{4} x^{4}+2 f w_{34} x^{4}-f_{4} w x^{3}+2 f w_{4} x^{3}}{4 f x^{3}}, \\
R_{1323}=-\frac{b\left(b f+f_{3} x+2 f\right)}{4 f x^{2}}, \quad R_{1324}=R_{1423}=-\frac{b f_{4}}{4 f x}, \\
R_{1414}=-\frac{-2 a b f_{3} r^{2}-2 a f_{3} r^{2}+f_{3} w_{3} x^{4}-f_{4} w_{4} x^{4}+2 f w_{44} x^{4}+f_{3} w x^{3}}{4 f x^{3}}, \\
R_{1424}=\frac{b f_{3}}{4 f x}, \quad R_{1434}=\frac{b^{2} f_{3} r}{4 f x^{2}}, \quad R_{3434}=-\frac{-f_{3}^{2}-f_{4}^{2}+f f_{33}+f f_{44}}{2 f}, \\
S_{11}=\frac{2 a^{2} f r^{2}-3 a b^{2} r^{2}-8 a b r^{2}+2 a f w x^{3}-6 a r^{2}-b\left(b w+w_{3} x+w\right) x^{3}-\left(w_{33}+w_{44}\right) x^{5}-2 w_{3} x^{4}}{-2 f x^{4}} \\
S_{12}=-\frac{2 a f-b^{2}-b}{2 f x^{2}}, \quad S_{44}=-\frac{b f f_{3}+f_{3}^{2} x+f_{4}^{2} x-f f_{33} x-f f_{44} x}{2 f^{2} x}, S_{34}=\frac{b f_{4}}{2 f x} \\
S_{13}=\frac{r\left(4 a f+b^{3}+b^{2}\right)}{2 f x^{3}}, \quad S_{33}=\frac{b^{2} f^{2}+2 b f^{2}+b f_{3} f x+f_{33} f x^{2}+f_{44} f x^{2}-f_{3}^{2} x^{2}-f_{4}^{2} x^{2}}{2 f^{2} x^{2}} \\
\kappa=\frac{-4 a f^{3}+b(3 b+4) f^{2}+2\left(f_{33}+f_{44}\right) f x^{2}-2\left(f_{3}^{2}+f_{4}^{2}\right) x^{2}}{2 f^{3} x^{2}}
\end{gathered}
$$

Again for zero cosmological constant, the non-zero components (upto symmetry) of the energy momentum tensor are given by

$$
\begin{gathered}
T_{11}=\frac{c^{4}}{32 \pi f^{3} G x^{4}} \quad\left[3 a b^{2} f^{2} r^{2}+12 a b f^{2} r^{2}+12 a f^{2} r^{2}+2 a f_{3}^{2} r^{2} x^{2}+2 a f_{4}^{2} r^{2} x^{2}-2 a f f_{33} r^{2} x^{2}\right. \\
-2 a f f_{44} r^{2} x^{2}-b^{2} f^{2} w x^{3}+2 b f^{2} w_{3} x^{4}-2 b f^{2} w x^{3}+2 f^{2} w_{33} x^{5}+2 f^{2} w_{44} x^{5} \\
\left.+4 f^{2} w_{3} x^{4}+2 f_{3}^{2} w x^{5}+2 f_{4}^{2} w x^{5}-2 f f_{33} w x^{5}-2 f f_{44} w x^{5}\right], \\
T_{12}=-\frac{c^{4}\left(b^{2} f^{2}+2 b f^{2}+2 f_{33} f x^{2}+2 f_{44} f x^{2}-2 f_{3}^{2} x^{2}-2 f_{4}^{2} x^{2}\right)}{32 \pi f^{3} G x^{2}}, \\
T_{13}=\frac{c^{4} r\left(4 a b f^{3}+8 a f^{3}+b^{3}\left(-f^{2}\right)-2 b^{2} f^{2}+2 b f_{3}^{2} x^{2}+2 b f_{4}^{2} x^{2}-2 b f f_{33} x^{2}-2 b f f_{44} x^{2}\right)}{32 \pi f^{3} G x^{3}}, \\
T_{33}=\frac{c^{4}\left(4 a f^{2}+b^{2}(-f)+2 b f_{3} x\right)}{32 \pi f G x^{2}}, \quad T_{34}=\frac{b c^{4} f_{4}}{16 \pi f G x}, \quad T_{44}=\frac{c^{4}\left(4 a f^{2}-3 b^{2} f-2 b f_{3} x-4 b f\right)}{32 \pi f G x^{2}} .
\end{gathered}
$$

From above we have the following:

Theorem 4.1. The pure radiation type metric (1.4) has the following curvature properties:

(i) It is a 3-quasi-Einstein manifold, since $S-\frac{b+b^{2}-2 a f}{2 f x^{2}} g$ is of rank 3.

(ii) For zero cosmological constant, its energy momentum tensor $T$ is of the form

$$
T=\alpha g+\beta_{1}\left(e_{1} \otimes e_{1}\right)+\beta_{3}\left(e_{3} \otimes e_{3}\right)+\beta_{4}\left(e_{4} \otimes e_{4}\right)+\sigma_{1}\left(e_{1} \otimes e_{3}+e_{3} \otimes e_{1}\right)+\sigma_{2}\left(e_{3} \otimes e_{4}+e_{4} \otimes e_{3}\right),
$$


where

$$
\begin{gathered}
e_{1}=\{1,0,0,0\}, \quad e_{3}=\{0,0,1,0\}, \quad e_{4}=\{0,0,0,1\}, \\
\alpha=-\frac{c^{4}\left[b(b+2) f^{2}+2\left(f_{33}+f_{44}\right) f x^{2}-2\left(f_{3}^{2}+f_{4}^{2}\right) x^{2}\right]}{32 \pi f^{3} G x^{2}}, \\
\beta_{1}=T_{11}-\alpha g_{11}, \quad \beta_{3}=T_{33}-\alpha g_{33}, \quad \beta_{4}=T_{44}-\alpha g_{44}, \quad \sigma_{1}=T_{13}-\alpha g_{13}, \quad \sigma_{2}=T_{34}-\alpha g_{34} .
\end{gathered}
$$

(iii) It is an Ein(4) manifold, such that

$$
\begin{aligned}
S^{4}= & \frac{\left(-2 a f+b^{2}+b\right)^{2}}{16 f^{8} x^{7}}\left[b(b+2) f^{3}\left(\left(f_{33}+f_{44}\right) x-b f_{3}\right)\right. \\
& +f^{2} x\left(\left(f_{33}+f_{44}\right)^{2} x^{2}-2 b(b+1)\left(f_{3}^{2}+f_{4}^{2}\right)\right) \\
& \left.-2\left(f_{3}^{2}+f_{4}^{2}\right)\left(f_{33}+f_{44}\right) f x^{3}+\left(f_{3}^{2}+f_{4}^{2}\right)^{2} x^{3}\right] g \\
- & \frac{\left(-2 a f+b^{2}+b\right)}{8 f^{7} x^{6}}\left[f^{4}\left(b^{2}(b+1)(b+2)-4 a\left(f_{33}+f_{44}\right) x^{2}\right)\right. \\
& +2 f^{3} x\left(x\left(2 a f_{4}^{2}+b(2 b+3)\left(f_{33}+f_{44}\right)\right)+2 a f_{3}^{2} x-(b+2) b^{2} f_{3}\right) \\
& -2 a b(b+2) f^{5}+2 f^{2} x^{2}\left(\left(f_{33}+f_{44}\right)^{2} x^{2}-3 b(b+1)\left(f_{3}^{2}+f_{4}^{2}\right)\right) \\
& \left.-4\left(f_{3}^{2}+f_{4}^{2}\right)\left(f_{33}+f_{44}\right) f x^{4}+2\left(f_{3}^{2}+f_{4}^{2}\right)^{2} x^{4}\right] S \\
+ & \frac{1}{4 f^{6} x^{4}}\left[4 a^{2} f^{6}+f^{4}\left(b^{2}(b+1)(3 b+5)-8 a\left(f_{33}+f_{44}\right) x^{2}\right)\right. \\
& +f^{3} x\left(x\left(8 a f_{4}^{2}+b(5 b+6)\left(f_{33}+f_{44}\right)\right)+8 a f_{3}^{2} x-(b+2) b^{2} f_{3}\right) \\
& -4 a b(2 b+3) f^{5}+f^{2} x^{2}\left(\left(f_{33}+f_{44}\right)^{2} x^{2}-6 b(b+1)\left(f_{3}^{2}+f_{4}^{2}\right)\right) \\
& \left.-2\left(f_{3}^{2}+f_{4}^{2}\right)\left(f_{33}+f_{44}\right) f x^{4}+\left(f_{3}^{2}+f_{4}^{2}\right)^{2} x^{4}\right] S^{2} \\
+ & \frac{1}{2 f^{3} x^{2}}\left[f^{2}(4 a f-b(3 b+4))+2\left(f_{3}^{2}+f_{4}^{2}-f\left(f_{33}+f_{44}\right)\right) x^{2}\right] S^{3},
\end{aligned}
$$

Remark 4.1. Since for zero cosmological constant $S=\frac{\kappa}{2} g+\frac{8 \pi G}{c^{4}} T$, hence from above theorem we can state that the Ricci tensor of (1.4) is of the form

$$
\begin{aligned}
S= & \left(S_{12}\right) g+\left(S_{11}-S_{12} g_{11}\right)\left(e_{1} \otimes e_{1}\right)+\left(S_{33}-S_{12} g_{33}\right)\left(e_{3} \otimes e_{3}\right)+\left(S_{44}-S_{12} g_{44}\right)\left(e_{4} \otimes e_{4}\right) \\
& +\left(S_{13}-S_{12} g_{13}\right)\left(e_{1} \otimes e_{3}+e_{3} \otimes e_{1}\right)+\left(S_{34}-S_{12} g_{34}\right)\left(e_{3} \otimes e_{4}+e_{4} \otimes e_{3}\right) .
\end{aligned}
$$

We can easilly check that $\left\|e_{1}\right\|=0$ and the nonzero components of $\nabla e_{1}$ are given by

$$
\nabla_{1} e_{1}=\frac{a r}{x^{2}}, \quad \nabla_{3} e_{1}=\frac{b}{2 x} .
$$

We know that a spacetime is called generalized pp-wave ([29], [35]) if there exists a covariantly constant null vector field. Hence we can state the following:

Theorem 4.2. The pure radiation type metric (1.4) represents generalized pp-wave if $a=b=0$. 
Now a spacetime is 2-quasi-Einstein if $\operatorname{Rank}(S-\alpha g)=0$. Hence the metric (1.4) becomes 2-quasiEinstein if one of the following condition holds

$$
\begin{gathered}
(i)\left(S_{34}-S_{12} g_{34}\right)=\left(S_{44}-S_{12} g_{44}\right)=0 \\
(i i)\left(S_{13}-S_{12} g_{13}\right)=\left(S_{33}-S_{12} g_{33}\right)=\left(S_{34}-S_{12} g_{34}\right)=0 \\
(\text { iii })\left(S_{11}-S_{12} g_{11}\right)=\left(S_{13}-S_{12} g_{13}\right)=0 .
\end{gathered}
$$

Simplifying the above conditions we can state the following:

Theorem 4.3. The pure radiation type metric (1.4) becomes 2-quasi-Einstein if any one the following condition holds

$$
\begin{aligned}
& \text { (i) } b=2 a f^{3}-\left(f_{3}^{2}+f_{4}^{2}-f\left(f_{33}+f_{44}\right)\right) x^{2}=0, \\
& \text { (ii) } f_{4}=2 a f^{3}+x f\left(x f_{33}-b f_{3}\right)-b(b+1) f^{2}-x^{2} f_{3}^{2}=0, \\
& \text { (iii) } a=b=-f_{3}^{2}+f_{4}^{2}+f\left(f_{33}+f_{44}\right)=0, \\
& \text { (iv) } f_{4}=a=b f_{3}+\frac{b f}{x}+x f_{33}-\frac{x f_{3}^{2}}{f}=0, \\
& \text { (v) } f_{4}=b-2=\frac{2 a f^{2}}{x}+x f_{33}-2 f_{3}-\frac{x f_{3}^{2}}{f}-\frac{2 f}{x}=0, \\
& \text { (vi) } a=(b+2) w_{3}+w_{33} x+w_{44} x=0, \\
& \text { (vii) } b-2=w_{33}+w_{44}=0 .
\end{aligned}
$$

Example 4.1. If we consider the metric (1.4) with $f(x, y)=\frac{e^{\frac{1}{3} x^{3}}}{x^{2 / 3}}, b=-2$ and $a=0$, then (1.4) becomes a 2-quasi-Einstein manifold.

Now a spacetime is perfect fluid if it satisfies (1.2). Hence the metric (1.4) represents perfect fluid if one of the following condition holds

$$
\begin{aligned}
& \text { (i) } \beta_{1}=\beta_{3}=\sigma_{1}=\sigma_{2}=0, \\
& \text { (ii) } \beta_{1}=\beta_{4}=\sigma_{1}=\sigma_{2}=0, \\
& \text { (iii) } \beta_{3}=\beta_{4}=\sigma_{1}=\sigma_{2}=0 .
\end{aligned}
$$

Simplifying the above conditions we can state the following:

Theorem 4.4. The pure radiation type metric (1.4) represents perfect fluid if any one the following condition holds

(i) $a=b=2 w_{3}+x w_{33}+x w_{44}=f_{3}^{2}+f_{4}^{2}-f\left(f_{33}+f_{44}\right)=0$,

(ii) $b+2=f_{4}=w_{33}+w_{44}=2 a f^{3}-x^{2} f_{3}^{2}+x f\left(x f_{33}-2 f_{3}\right)-2 f^{2}=0$,

(iii) $a=f_{4}=(b+2) w_{3}+w_{33} x+w_{44} x=x f\left(b f_{3}+x f_{33}\right)+b f^{2}-x^{2} f_{3}^{2}=0$,

(iv) $a=f_{4}=x f\left(b f^{\prime}+x f^{\prime \prime}\right)+b f^{2}-x^{2} f^{\prime 2}=x f\left(b f^{\prime}-x f^{\prime \prime}\right)+b(b+1) f^{2}+x^{2} f^{\prime 2}=0$,

(v) $b+2=f-\frac{1}{a}=0$. 
Example 4.2. Consider the metric (1.4), where $w(u, x, y)=u x y, f(x, y)=\frac{e^{\frac{1}{3} x^{3}}}{x^{2 / 3}}, b=-2$ and $a=0$, then (1.4) represents a perfect fluid spacetime. The energy momentum tensor of this metric can be expressed as

$$
T=-\frac{c^{4} e^{-\frac{x^{3}}{3}}\left(3 x^{3}+1\right)}{24 \pi G x^{4 / 3}} g+\frac{c^{4}\left(3 x^{3}-2\right)}{12 \pi G x^{2}}\left(e_{4} \otimes e_{4}\right) .
$$

Moreover in this case the metric is quasi-Einstein and $\operatorname{Ein}(2)$.

Again a spacetime is a pure radiation spacetime if it satisfies (1.1). Now $\left\|e_{1}\right\|=0$ and hence the metric (1.4) represents perfect fluid if $\alpha=\beta_{3}=\beta_{4}=\sigma_{1}=\sigma_{2}=0$. Simplifying these conditions we can state the following:

Theorem 4.5. The pure radiation type metric (1.4) represents pure radiation if $f \equiv \frac{1}{a}$ and $b=-2$.

Again from above we can easily calculate (large but straightforward) the components of $R \cdot R$, $Q(g, R)$ and $Q(S, R)$. Then we have the following:

Theorem 4.6. The pure radiation type metric (1.4) is

(i) Ricci generalized pseudosymmetric $(R \cdot R=Q(S, R))$ if $b=-2$ and $f$ is constant. And in this case the metric is $R$-space by Venzi for the associated 1-form $\{1,0,0,0\}$,

(ii) a manifold of vanishing scalar curvature if $f \equiv \frac{b(3 b+4)}{4 a}$,

(iii) semisymmetric if $b=-2$ and $f \equiv \frac{1}{a}$.

From (1.3), (1.4) and (1.5), we see that both the pure radiation metric and the pp-wave metric are special cases of the metric (1.4). We refer the reader to see [26], [43] and also references therein for recent works on pp-wave metric. We now draw a comparison (similarities and dissimilarities) between the curvature properties of pure radiation metric and pp-wave metric.

\section{A. Similarities:}

(1) Both the metrics are of vanishing scalar curvature,

(2) both are $R$-space by Venzi as well as $C$-space by Venzi,

(3) both are semisymmetric and semisymmetric due to conformal curvature tensor,

(4) for both the metrics $Q(S, R)=Q(S, C)=0$,

(5) both the metrics are Ricci simple,

(6) Ricci tensors of both metrics are Riemann compatible as well as conformal compatible,

(7) Ricci 1-forms $\Lambda_{(Z) l}$ of both metrics are recurrent,

(8) conformal 2-forms $\Omega_{(C) l}^{m}$ of both metrics are recurrent,

(9) for both the metrics $P \cdot R=0$ but $P \cdot \mathcal{R} \neq 0$.

(10) both are weakly Ricci symmetric and hence weakly cyclic Ricci symmetric,

(11) both the metrics satisfy $P \cdot P=-\frac{1}{3} Q(S, P)$, 
(12) the energy-momentum tensors of both the metrics are semisymmetric.

\section{B. Dissimilarities:}

(1) For zero cosmological constant, the energy-momentum tensors of both the metrics are of rank one, but the associated 1-form for radiation metric is null, and for pp-wave metric it is null as well as covariantly constant,

(2) pp-wave metric is Ricci recurrent but pure radiation metric is not so,

(3) for pp-wave metric energy-momentum tensor is cyclic parallel if and only if it is parallel but this fact is not true for pure radiation metric.

\section{REFERENCES}

[1] Adamów, A. and Deszcz, R., On totally umbilical submanifolds of some class of Riemannian manifolds, Demonstratio Math., 16 (1983), 39-59.

[2] Besse, A.L., Einstein Manifolds, Springer-Verlag, Berlin, Heidelberg, 1987.

[3] Brinkmann, H. W., Einstein spaces which are mapped conformally on each other, Math. Ann., 94 (1925), 119-145.

[4] Cartan, É., Sur une classe remarquable d'espaces de Riemannian, Bull. Soc. Math. France, 54 (1926), $214-264$.

[5] Cartan, É., Leçons sur la géométrie des espaces de Riemann, 2nd ed., Paris, 1946.

[6] Chaki, M.C., On pseudosymmetric manifolds, An. Ştiinţ. ale Univ., AL. I. Cuza din Iaşi N. Ser. Sect. Ia, 33(1) (1987), 53-58.

[7] Chaki, M. C., On pseudo Ricci symmetric manifolds, Bulgarian J. Phys., 15 (1988), 526-531.

[8] Deszcz, R., On pseudosymmetric spaces, Bull. Belg. Math. Soc., Ser. A, 44 (1992), 1-34.

[9] Deszcz, R. and Głogowska, M., Some examples of nonsemisymmetric Ricci-semisymmetric hypersurfaces, Colloq. Math., 94 (2002), 87-101.

[10] Deszcz, R., Głogowska, M., Hotloś, M. and Șentürk, Z., On certain quasi-Einstein semi-symmetric hypersurfaces, Ann. Univ. Sci. Budapest Eötvös Sect. Math., 41 (1998), 151-164.

[11] Deszcz, R., Głogowska, M., Jełowicki, L., Petrović-Torgašev, M. and Zafindratafa, G., On Riemann and Weyl compatible tensors, Publ. Inst. Math. (Beograd) (N.S.), 94(108) (2013), 111-124.

[12] Deszcz, R., Głogowska M, Hotloś M and Sawicz K. A Survey on Generalized Einstein Metric Conditions, Advances in Lorentzian Geometry, Proceedings of the Lorentzian Geometry Conference in Berlin, AMS/IP Studies in Advanced Mathematics, 49, S.-T. Yau (series ed.), M. Plaue, A.D. Rendall and M. Scherfner (eds.), 2011, 27-46.

[13] Deszcz, R., Haesen, S. and Verstraelen, L., On natural symmetries, Chapter 6 in "Topics in Differential Geometry", Editors A. Mihai, I. Mihai and R. Miron, Editura Acad. Române (2008).

[14] Deszcz, R. and Hotloś, M., On hypersurfaces with type number two in spaces of constant curvature, Ann. Univ. Sci. Budapest Eötvös Sect. Math., 46 (2003), 19-34.

[15] Deszcz, R., Hotloś, M., Jełowicki, J., Kundu, H. and Shaikh, A.A., Curvature properties of Gödel metric, Int J. Geom. Method Mod. Phy., 11 (2014), 20 pages.

[16] Gray, A., Einstein-like manifolds which are not Einstein, Geom. Dedicta, 7 (1978), 259-280.

[17] Głogowska, M., Semi-Riemannian manifolds whose Weyl tensor is a Kulkarni-Nomizu square, Publ. Inst. Math. (Beograd) (N.S.), 72(86) (2002), 95-106.

[18] Ishii, Y., On conharmonic transformations, Tensor (N. S.), 11 (1957), 73-80. 
[19] Lovelock, D. and Rund, H., Tensors, differential forms and variational principles, Courier Dover Publications, 1989.

[20] Ludwig, G. and Edgar, S. B., Conformally Ricci flat pure radiation metrics, Class. Quant. Grav., 14(12) (1997), 3453-3473.

[21] Mantica, C. A. and Molinari, L. G., Riemann compatible tensors, Colloq. Math., 128 (2012), 197-210.

[22] Mantica, C. A. and Molinari, L. G., Weyl compatible tensors, Int. J. Geom. Meth. Mod. Phys., 11(08) (2014), 1450070, (20 pages).

[23] Mantica, C. A. and Suh, Y. J., The closedness of some generalized curvature 2-forms on a Riemannian manifold I, Publ. Math. Debrecen, 81/3-4 (2012), 313-326.

[24] Mantica, C. A. and Suh, Y. J., The closedness of some generalized curvature 2-forms on a Riemannian manifold II, Publ. Math. Debrecen, 82/1 (2013), 163-182.

[25] Mantica, C. A. and Suh, Y. J., Recurrent conformal 2-forms on pseudo-Riemannian manifolds, Int. J. Geom. Meth. Mod. Phy., 11(6) (2014), 1450056 (29 pages).

[26] Mantica, C. A. and Suh, Y. J., Pseudo-Z symmetric space-times with divergence-free Weyl tensor and pp-waves, Int. J. Geom. Meth. Mod. Phys., 13(02) (2016), 1650015.

[27] Patterson, E. M., Some theorems on Ricci recurrent spaces, J. London Math. Soc., 27 (1952), 287-295.

[28] Prvanović, M., On weakly symmetric Riemannian manifolds, Publ. Math. Debrecen, 46(1-2) (1995), $19-25$.

[29] Radhakrishna, L. and Singh, N. I., Pure radiation fields admitting nontrivial null symmetries, J. Math. Phys., 25 (1984), 2293-2300, doi: 10.1063/1.526434.

[30] Ruse, H. S., On simply harmonic spaces, J. London Math. Soc., 21 (1946), 243-247.

[31] Ruse, H. S., On simply harmonic 'kappa spaces' of four dimensions, Proc. London Math. Soc., 50 (1949), 317-329.

[32] Ruse, H. S., Three dimensional spaces of recurrent curvature, Proc. London Math. Soc., 50 (1949), 438-446.

[33] Shaikh, A. A., Al-Solamy, F. R. and Roy, I., On the existence of a new class of semi-Riemannian manifolds, Mathematical Sciences, 7:46 (2013), 1-13.

[34] Shaikh, A. A., Ali, M. and Ahsan, Z., Curvature properties of Robinson-Trautman metric, arXiv:1701.06136v1 [math.DG] 22 Jan 2017.

[35] Shaikh, A. A., Binh,T. Q. and Kundu, H., Curvature properties of generalized pp-wave metric, arXiv:1702.07497v1 [math.DG] 24 Feb 2017.

[36] Shaikh, A.A., Deszcz, R., Hotloś, M., Jełowicki, J. and Kundu, H., On pseudosymmetric manifolds, Publ. Math. Debrecen, 86(3-4) (2015), 433-456.

[37] Shaikh, A. A. and Kundu, H., On weakly symmetric and weakly Ricci symmetric warped product manifolds, Publ. Math. Debrecen, 81(3-4) (2012), 487-505.

[38] Shaikh, A. A. and Kundu, H., On equivalency of various geometric structures, J. Geom., 105 (2014), 139-165, DOI: $10.1007 / \mathrm{s} 00022-013-0200-4$.

[39] Shaikh, A. A. and Kundu, H., On generlized Roter type manifolds, arXiv:1411.0841v1 [math.DG] 4 Nov 2014.

[40] Shaikh, A. A. and Kundu, H., On curvature properties of Som-Raychaudhuri spacetime, J. Geom., DOI: 10.1007/s00022-016-0355-x.

[41] Shaikh, A. A. and Kundu, H., On some curvature restricted geometric structures for projective curvature tensor, arXiv:1609.04749v1 [math.DG], 15 Sep 2016.

[42] Shaikh, A. A. and Kundu, H., On warped product manifolds satisfying some pseudosymmetric type conditions, arXiv:1612.03297v1 [math.DG], 10 Dec 2016.

[43] Shaikh, A. A. and Kundu, H., Curvature properties of generalized pp-wave metric, Preprint. 
[44] Shaikh, A. A., Kundu, H. and Ali, Md. S., On warped product super generalized recurrent manifolds, To appear in An. Ştiinţ. Univ. Al. I. Cuza Iaşi Mat. (N. S.).

[45] Shaikh, A.A. and Jana S.K., On weakly cyclic Ricci symmetric manifolds, Ann. Pol. Math., 89(3) (2006), $139-146$.

[46] Shaikh, A. A. and Patra, A., On a generalized class of recurrent manifolds, Arch. Math. (BRNO), 46 (2010), 71-78.

[47] Shaikh, A. A. and Roy, I., On weakly generalized recurrent manifolds, Ann. Univ. Sci. Budapest, Eötvös Sect. Math., 54 (2011) 35-45.

[48] Shaikh, A. A., Roy, I. and Kundu, H., On the existence of a generalized class of recurrent manifolds, arXiv:1504.0253v1 [math.DG] 10 Apr 2015.

[49] Shaikh, A. A., Roy, I. and Kundu, H., On some generalized recurrent manifolds, Bull. Iranian Math. Soc., 43(5) (2017).

[50] Stephani. H., Kramer, D., Maccallum, M., Hoenselaers, C. and Herlt, Exact Solutions of Einstein's Field Equations, 2nd Ed., Cambridge Univ. Press, UK, 2003.

[51] Szabó, Z.I., Structure theorems on Riemannian spaces satisfying $R(X, Y) \cdot R=0$, I. The local version, J. Diff. Geom., 17 (1982), 531-582.

[52] Szabó, Z. I., Classification and construction of complete hypersurfaces satisfying $R(X, Y) \cdot R=0$, Acta Sci. Math., 47 (1984), 321-348.

[53] Szabó, Z. I., Structure theorems on Riemannian spaces satisfying $R(X, Y) \cdot R=0$, II, The global version, Geom. Dedicata, 19 (1985), 65-108.

[54] Suh, Y. J., Kwon, J-H. and Pyo, Y. S., On semi-Riemannian manifolds satisfying the second Bianchi identity, J. of Korean Math. Soc., 40(1) (2003), 129-167.

[55] Támassy, L. and Binh, T.Q., On weakly symmetric and weakly projective symmetric Riemannian manifolds, Coll. Math. Soc. J. Bolyai, 50 (1989), 663-670.

[56] Tamássy, L. and Binh, T. Q., On weak symmetries of Einstein and Sasakian manifolds, Tensor (N. S.), 53 (1993), $140-148$.

[57] Venzi, P., Una generalizzazione degli spazi ricorrenti, Revue Roumaine de Math. Pure at appl., 30 (1985), 295-305.

[58] Walker, A.G., On Ruse's spaces of recurrent curvature, Proc. London Math. Soc., 52 (1950), 36-64.

[59] Wils, P., Homogeneous and conformally Ricci flat pure radiation fields, Class. Quant. Grav., 6(9) (1989), $1243-1251$.

[60] Yano, K. and Kon, M., Structures on manifolds, World Scientific Publ., Singapore, 1989.

1,3 Department of Mathematics, Aligarh Muslim University,

Aligarh-202002,

Uttar Pradesh, India

E-mail address: aask2003@yahoo.co.in, aashaikh@math.buruniv.ac.in

E-mail address: musavvirali.maths@amu.ac.in

2 Department of Mathematics,

University of Burdwan, Golapbag,

BURDWAN-713104,

West Bengal, India

E-mail address: kundu.haradhan@gmail.com 
${ }^{4}$ Faculty of Science And Technology, University of ISLAMiC SCIENCES, Nilai, Malaysia

E-mail address: zafar.ahsan@rediffmail.com 